\title{
TERAPÊUTICA E PROFILAXIA DA MALÁRIA. REVISÃo
}

\author{
João Guimarães de Andrade*
}

\section{RESUMO}

Não há perspectivas previsíveis de controle nem de erradicação da malária nos próximos anos. A antiga estratégia de combate ao vetor, tem-se mostrado pouco efetiva no decorrer dos anos e a vacina da malária apesar de resultados promissores de estudos preliminares conduzidos em campo, necessita ainda ser melhor avaliada. Assim, a estratégia atual de controle da malária consiste no tratamento e prevenção. Nesta revista serão abordados aspectos práticos sobre o manuseio das drogas antimaláricas, em especial aquelas utilizadas para o Plasmodium falciparum. A cloroquina continua sendo a droga de escolha para o $P$. ovale, $P$. malarie $P$. vivax. A escolha de uma droga para o $P$. falciparum vai depender da sensibilidade prévia conhecida de uma determinada droga naquele país ou naquela região. Mesmo os mais novos medicamentos como a mefloquina e o halofantrine, é crescente o desenvolvimento da resistência. A grande expectativa em relação aos derivados da artemisia parece estar se confirmando em ensaios clínicos. Atualmente a tetraciclina associada ao quinino tem mostrado os maiores índices de cura para a terapêutica da malária por $P$. falciparum, no Brasil e poderia ser a droga de escolha. A mefloquina e mesmo as mais novas drogas, se disponíveis, poder , ao ser recomendadas se houver falha terapêutica ou intolerância à associaç ,ao de quinino e tetraciclina. A ex-sanguíneo transfus , ao pode ser uma medida adicional em situaçøes especiais, tais como mais que $10 \%$ de eritrócitos parasitados e na malária cerebral. Na gravidez, a malária por falciparum deve ser tratada sem maiores receios com quinino. Enfatiza-se a grande necessidade de novos compostos, cuja estrutura química não seja relacionada aos antimaláricos já disponíveis. Estas novas drogas deveriam ser usadas com cautela, para minimizar o risco de emergência de resistência.

\section{UNITERMOS: Malária. Plasmodium falciparum. Plasmodium malarie.} Plasmodium vivax. Resistência a drogas. Profilaxia. Terapêutica.

A malária é a mais importante de todas as doenças tropicais. Anualmente, cerca de duzentos milhões de pessoas adquirem malária em todo o mundo e ocorrem 2 milhões de mortes, principalmente crianças com idades abaixo de 5 anos. A grande esperança de erradicação da malária na década de $60 \mathrm{com}$ base, principalmente, no combate ao vetor, não se tornou realidade, acometendo agora mais pessoas do que naquele período. Por outro lado, apesar de resultados promissores obtidos com a vacina

Prof. do Depto. de Medicina Tropical, Instituto de Patologia Tropical e Saúde Pública e do Hospital de Doenças Tropicais. Endereço para correspondência: Departamento de Medicina Tropical, Hospital das Clínicas. Primeira Avenida s/n, Setor Universitário, Caixa Postal 131, Goiânia, Goiás, Brasil.

Recebido para publicação em 10/05/94 
\begin{tabular}{llllllll}
\hline ANDRADE,J.G. Terapêutica & e & Profilaxia & da & Malária.Revisão.Rev.Pat.Trop.23 & ( & 2 & ):181-
\end{tabular} 190.jul./dez.1994

da malária em estudos de intervenção conduzidos em campo, não se vislumbra, a curto prazo, a utilização de vacinas antimaláricas no controle da doença ${ }^{21,27}$. Novos estudos onde se avalie o efeito protetor, assim como a análise custo-efetividade, serão fundamentais e necessários para que se possa empregá-la como rotina nos programas de controle.

A ampla disseminação e o rápido aumento e propagação da resistência do Plasmodium falciparum às drogas antimaláricas é sistematicamente progressivo de um país a outro, contribuindo sobremaneira para as dificuldades de controle e erradicação 7,16,17. As dimensões continentais do Brasil, facilitando os movimentos migratórios principalmente em direção aos garimpos e novas fronteiras agrícolas localizados na Bacia Amazônica, onde se concentra mais de $90 \%$ dos casos de malária no Brasil, favorece a disseminação da resistência. As regiões ainda livres de resistência estão limitadas à América Central e partes do oeste da África e Ásia, mas um avanço da resistência num futuro próximo para estas regiões é esperado ${ }^{12}$. Isto se deve não apenas a uma notável adaptação do parasito, mas também ao uso abusivo, indiscriminado e incorreto das drogas com finalidades profilática e terapêutica ${ }^{17}$

Também, o uso quase rotineiro de antimaláricos em pacientes febris, especialmente crianças de áreas endêmicas, favorecem o aumento da resistência. fenômeno de "pressão continuada" de uma determinada droga parece ser fator fundamental ao desenvolvimento da resistência.

Há importantes considerações que merecem ser discutidas: 1 - o diagnóstico da malária requer um alto índice de suspeição, porque a apresentação clínica nem sempre é típica, principalmente em pacientes semi-imunes ou que tenham usado drogas antimaláricas previamente; 2- em virtude da infecção por $P$. falciparum ser potencialmente grave e progredir rapidamente para complicações, especialmente em pacientes não imuunes, há necessidade de um diagnóstico rápido para instituição precoce da terapêutica. É aconselhável que o tratamento seja supervisionado e de preferência que o paciente seja hospitalizado; 3 - a escolha da droga, deve basear-se no conhecimento do padrão atual de sensibilidade das cepas do P. falciparum; 4 - a resposta clínica e parasitológica deve ser monitorada cuidadosamente para detecção de falha terapêutica; 5 - monitoração contínua para detecção de complicações é essencia na malária cau sada por $P$. falciparum, pois poderá ser necessário corrigí-las com terapia apropriada.

Se o diagnóstico de malária por $P$. falciparum puder ser excluído com segurança, o paciente deverá receber cloroquina, de preferência por via oral, pois o $P$. ovale, P.malarie e P vivax são uniformemente sensíveis a esta droga ${ }^{1,29}$. O tratamento pode ser realizado ambulatorialmente na maioria das vezes. A defervecência dos sintomas e parasitemia são esperados em $72 \mathrm{hs}$ após o início da terapia. Para pacientes incapazes de reter a droga oral ou pacientes graves (geralmente causada por \begin{tabular}{llllllll}
\hline ANDRADE,J.G. & Terapêutica & e & Profilaxia & da & Malária.Revisão.Rev.Pat.Trop.23 ( 2 ):181-
\end{tabular} 190,jul./dez.1994

P.falciparum) a via parenteral deve ser preferida (Tabela 1). Para a prevenção de relapsos de malária causados por P.vivax, P.malarie e $P$. ovale, adquiridas por picada de mosquito, administrar primaquina na dosagem de $15 \mathrm{mg} / \mathrm{dia}$, por 14 dias. Na malária transmitida pelo sangue (transfusional, acidente de laboratório, etc) como não ocorre ciclo esquizogônico hepático, não há necessidade de usar medicação preventiva. Pacientes que adquirem malária por P.falciparum em regiões da América Central, Haiti e Oriente Médio, podem ser tratados também com cloroquina. (TABELA 1 ).

Tabela 1. Quimioterapia e quimioprofilaxia recomendadas para malária*

\begin{tabular}{|c|c|c|c|}
\hline Indicação & Droga & Via & Dosagem $^{* *}$ \\
\hline Malária não complicada & Cloroquina & VO & $\begin{array}{l}10 \mathrm{mg} / \mathrm{kg}, 5 \mathrm{mg} \text { após } 6 \mathrm{hs} \text { e } 5 \mathrm{mg} \\
\text { no } 2^{\circ} \text { e } 3^{\circ} \mathrm{dia}\end{array}$ \\
\hline Malária não complicada & Cloroquina & IM,EV & Mesma, como acima \\
\hline Infecção grave & $\begin{array}{l}\text { Cloroquina } \\
\text { ou }\end{array}$ & EV & Mesma, como acima \\
\hline Ouiminnrofilayia & Quinino & EV & $25 \mathrm{mg} / \mathrm{kg} / \mathrm{d}, 3 \mathrm{x}, 7-10$ dias \\
\hline Quimioprofilaxıa & Cloroquina & $\mathrm{VO}$ & $5 \mathrm{mg} / \mathrm{kg} / \mathrm{s}$ \\
\hline
\end{tabular}

Adaptado de American Public Health Association, Control of Comunicable Disease in Man, fifteenth edition, 1990

* Todas as espécies de plasmodium exceto $P$. falciparum cloroquino-resistente.

** d significa dose diária e s significa dose semanal.

A terapêutica da malária por Plasmodium falciparum adquirida em todas as outras regiões do mundo, deveriam ser consideradas resistentes à cloroquina e isto é mais complexo e depende obviamente da sensibilidade prévia conhecida de uma determinada droga, na região. Esquemas terapêuticos importados de outros países não devem ser utilizados, pois mesmo dentro de um país a sensibilidade do $P$.falciparum às drogas antimaláricas varia de região para região, e mesmo na própria região ${ }^{11}$. O que se deve ter em mente ao lidar com pacientes com malária é o caráter dinâmico da sensibilidade do $P$. falciparum, onde o desenvolvimento de resistência é uma possibilidade em potencial, e que mais cedo ou mais tarde o $P$. falciparum desenvolverá resistência a qualquer nova droga atual ou em desenvolvimento.

Várias tentativas têm sido realizadas para restaurar a eficácia da cloroquina, uma droga de baixo custo, universalmente disponível, rápida de ação e de poucos efeitos colaterais. À semelhança do que ocorre com os antibióticos, a descontinuidade 
do uso da cloroquina poderia reverter a sensibilidade perdida. $\mathrm{Na}$ Tailândia, tal fenômeno parece estar ocorrendo ${ }^{20}$. Esta estratégia entretanto, necessitaria ser ampla, interessando a todos os países e regióes onde tenha ocorrido qualquer tipo de resistência à cloroquina.

Outros estudos têm mostrado evidências de reversão da sensibilidade do $P$. falciparum à cloroquina, utilizando bloqueadores de canais de cálcio e tricíclicos antidepressivos ${ }^{3,10,16}$. Este é um campo de investigação que pode ser promissor para 0 futuro da terapia antipalúdica. Tem sido desapontante a utilização de cloroquina em altas doses, pois, apesar do rápido clearance da parasitemia, o aparecimento de resistência tardia tipo R1 é muito comum ${ }^{2}$. Ao que parece, estas tentativas serão insuficientes para reverter a sensibilidade perdida da cloroquina, a ponto de se poder reutilizá-la em larga escala na terapêutica da malária por $P$.faciparum.

Os resultados de vários trabalhos realizados no Brasil, mostrando o alto grau de resistência do $P$. falciparum à cloroquina, nos induz a afirmar que não há mais lugar para a utilização desta droga no tratamento da malária causada pelo $P$. falciparum $2,13,14,18$. Seu uso continuado só contribuirá para a disseminação da malária resistente. Também, a utilização dos sulfamídicos isoladamente, certamente não é aconselhável, devido ao alto nível de resistência adquirida nos últimos anos ${ }^{13,18}$

Assim, para a malária acusada por $P$. falciparum resistente não complicada cujo o paciente possa reter o medicamento por via oral, o sulfato de quinino como droga única ou associado a outras drogas será a medicação de escolha (Tabela 2). Atualmente, no Brasil, a melhor opção é a associação de sulfato de quinino tetraciclina, devido a sua eficácia, disponibilidade, segurança e baixo custo ${ }^{13,18,23}$. Do ponto de vista de Saúde Pública, a necessidade de fracionamento das doses diárias, e o tempo prolongado de uso são fatores limitantes e indesejáveis.

Para a malária causada pelo $P$. falciparum resistente não complicada onde não se possa administrar o medicamento por via oral, e em casos de malária grave, o dihydroclorato de quinino por via venosa deve ser a droga de preferência (Tabela 2).

A mefloquina por via oral e em dose única tem sido utilizada por diversos anos, em larga escala no sudeste da Ásia, sob controle na América Latina e, raramente, na África. $\mathrm{O}$ crescente aumento da resistência, ambos, in vitro e in vivo, elevado custo, e a possibilidade de produzir reação neuropsíquica de moderada a grave intensidade, com freqüuência não desprezível, faz com que sua utilização seja restrita ${ }^{8,16,19}$.

A eficácia do halofantrine e dos derivados do qinghaosu (um extrato da planta Artemisia annua) tem sido observada em diversos estudos, mas altas taxas de recaída são freqüentes ${ }^{8,9,24,25,28}$. A artemisina promove mais rápido clearance da parasitemia fato ainda não observado em outras drogas antimaláricas disponíveis, parecendo ser uma droga importante, talvez a mais indicada em pacientes com malária por P.falciparum na sua forma grave com alta parasitemia, e na malária cerebral, onde a velocidade de clearance parasitêmico é muito importante e fundamental. Deve ser enfatizada a possibilidade de resistência cruzada entre mefloquina, quinino e halofantrine, situação que preocupa os pesquisadores e profissionais que lidam com a malária.

Tabela 2. Quimioterapia e quimioprofilaxia recomendadas para malária causada por Plasmodium falciparum resistente.

\begin{tabular}{|c|c|c|c|}
\hline Indicação & Droga & Via & Dosagem \\
\hline \multirow[t]{5}{*}{$\begin{array}{l}\text { Malária } \\
\text { complicada }\end{array}$} & Quinino & VO & $\begin{array}{l}25 \mathrm{mg} / \mathrm{kg} / \mathrm{dia}, \quad 3 \mathrm{x}, \quad 7-10 \\
\text { dias }\end{array}$ \\
\hline & $\begin{array}{l}\text { Quinino } \\
+\end{array}$ & VO & Mesma, como acima \\
\hline & Pirimetamina- sulfadiazina & VO & 3 tabletes \\
\hline & $\begin{array}{l}\text { Quinino } \\
+\end{array}$ & VO & $25 \mathrm{mg} / \mathrm{kg} / \mathrm{dia}, 3 \mathrm{x}, 3$ dias \\
\hline & Tetraciclina & VO & $1 \mathrm{gr} / \mathrm{dia}, 4 \mathrm{x}, 7 \mathrm{dias}$ \\
\hline $\begin{array}{l}\text { Malária não } \\
\text { complicada }\end{array}$ & Quinino & ,EV & $25 \mathrm{mg} / \mathrm{kg} / \mathrm{dia}, 3 \mathrm{x}$ \\
\hline $\begin{array}{l}\text { Infecção grave } P \text {. } \\
\text { falciparum }\end{array}$ & Quinino & EV & Mesma, como acima \\
\hline Quimioprofilaxia & $\begin{array}{l}\text { Mefloquina } \\
\text { Doxiciclina } \\
\text { Sulfadoxina-Pirimetamina }\end{array}$ & $\begin{array}{l}\text { VO } \\
\text { VO } \\
\text { VO }\end{array}$ & $\begin{array}{l}250 \mathrm{mg} / \mathrm{s} \\
100 \mathrm{mg} / \mathrm{d} \\
2 \text { tabletes } / \mathrm{s}\end{array}$ \\
\hline
\end{tabular}

Informaçðes adaptadas de American Public Health Association, Control of Comunicable Disease in Man, fifteenth edition, 19901

Uma questão ainda não resolvida, é o uso de ex-sanguíneo transfusão, para casos graves de malária causada por P.falciparum. A despeito da falta de estudos randomizados e controlados demonstrando a eficácia da ex-sanguíneo transfusão, este procedimento parece ser útil como medida adjuvante em determinados grupos de pacientes 6,7. Várias questões ainda estão indefinidas, incluindo as indicações específicas, volume da ex-sanguíneo e a escolha do produto a ser infundido; se sangue total ou apenas eritrócitos. Tendo em mente estas considerações, a ex-sanguíneo transfusão para tratamento de malária grave por falciparum, poderia ser considerada nas seguintes situações: eritrócitos circulantes com parasitemia $>10 \%$; malária cerebral; 
coagulação intravascular disseminada e insuficiência renal aguda. O mau prognóstico de pacientes idosos com malária por falciparum, faz com que se possa ser mais liberal na indicação da ex-sanguíneo, nesta população em especial.

A terapêutica da malária na gravidez merece considerações especiais. A cloroquina durante a gravidez é considerada segura para a mãe e para o feto. Recentes estudos indicam que a antiga preocupação de que o quinino causaria aborto, são infundadas. É unânime a opinião entre os malariologistas de que os riscos de complicações da malária na gravidez, superam qualquer risco teórico de efeito tóxico do quinino. As tetraciclinas devem ser evitadas, pois, interfere com o crescimento ósseo e coloração dos dentes. Os antifolatos apesar de potencialmente teratogênicos, são relativamente seguros na gravidez. Os sulfamídicos podem causar kernícterus, porisso devem ser evitados no terceiro trimestre da gravidez. A clindamicina é segura na gravidez, mas não há estudos relevantes de sua eficácia na gestação. Entretanto, constitui-se em uma alternativa adicional para a terapêutica de malária causada por cepas de P.falciparum resistentes ou intolerantes a outras drogas.

A terapêutica inespecífica é freqüentemente necessária em casos de malária grave por $P$. falciparum. A hipoglicemia é relativamente comum, especialmente em grávidas e em pacientes com malária cerebral, podendo ser precipitada ou piorada pela administração de quinino ${ }^{26}$. Deve ser constantemente monitorada e corrigida o mais rápido possível. A anemia, se importante, deve ser corrigida de preferência com concentrado de glóbulos vermelhos. Os corticosteróides não devem ser utilizados na malária cerebral, pois nao trazem benefício e podem ser nocivos ${ }^{22}$. Complicações ocasionais como a septicemia e o choque séptico, causados por gram negativos, devem merecer vigilância pela gravidade e possibilidade de serem confundidas com a própria malária.

A necessidade de profilaxia para qualquer espécie de plasmodium, deve ser decidida individualmente, caso a caso, na dependência de vários fatores: tempo de demora na viagem, intinerário, estação do ano, maneira de viajar, tipo de acomodações e fundanentalmente dos aspectos epidemiológicos da região para onde o indivíduo se dirige. Nenhuma recomentadação uniforme é possível. A combinação de proteção pessoal e quimioprofilaxia quando empregada de maneira apropriada é altamente efetiva. As seguintes medidas pessoais deveriam ser instiuidas; uso de camisas de mangas compridas e calças longas, aplicação de inseticidas repelentes, ar condicionado ou ven:ilador no quarto de dormir, redes de boa qualidade e de preferência impregradas com inseticidas, cortinados de malhas finas e uso de mosquiteiros. Decidindo-se pela profilaxia medicamentosa, esta deve ser iniciada uma semana antes da partida para zona endêmica, manutenção durante a permanência e até 4 semanas após deixá-la. Nas tabelas 1 e 2 são mostradas as drogas recomendadas pua a profilaxia, na dependência da sensibilidade das cepas de plasmodium que potencialmente possam estar envolvidas. Parece claro que a doxiciclina ou mefloquina devam ser as drogas mais indicadas na profilaxia da malária onde a resistência do $P$. falciparum seja importante, tal como o Brasil.

Finalmente, deve ser enfatizada a crescente expectativa de novas associações de drogas, ainda em fase de investigação, como mefloquina com sulfadoxina mais pirimetamina, e mefloquina com tetraciclina ${ }^{4,5}$.

Assim, há grande necessidade de novas drogas, com eficácia não comprometida pela resistência. Estas drogas deveriam ser usadas com cuidados, para diminuir o risco de induzir resistência. Uma droga antimalárica ideal e que se espera para o futuro deverá satisfazer as seguintes expectativas: bem tolerada, efetiva contra todas as espécies de plasmodium, dose única, disponível para uso em crianças, disponível em formulação oral e parenteral, nova estrutura química não relacionada aos antimaláricos atualmente existentes e de baixo custo. A disponibilidade de novas drogas com meia vida curta, diminuiria a chance de desenvolvimento de resistência por reinfecção.

\section{SUMMARY}

\section{Treatment and Prophylaxis of the Malaria . Revision}

There is still no immediate prospect of substantial improvement on control and possibility of erradication of malaria. The old strategy to reduce malaria transmission by vector control has failed, and nowadays the hope is towards the vaccine. However, the vaccine is not available yet, so that treatment and preventive measures are the only accessible tools up to now. In this review the main approach will be on antimalarial falciparum drugs. The drug choice to Plasmodium ovale, $P$. malariae and $P$. vivax lasts to be the chloroquine. Thus, to $P$. falciparum the drug choice depends on the sensitivity of the strains in that region or country. There is an increased resistance to novel drugs such as mefloquine and halofantrine, but the arthemisin is a great hope to the future. Nowadays, the best drug for treating malaria falciparum in Brazil is quinine plus tetracicline. Exchange transfusion is a controversial additional measure used i.e., in cerebral malaria and when eritrocytes parasited are grater than $10 \%$. Pregnant women should receive quinino, because there is no real evidence it may cause damage. Thus, there is a great need for new drugs with no compromised efficacy by drug resistance. Such drugs must to be used with caution and care in order minimize the risk of emerging resistance.

KEY WORDS: Malaria. Drugs resistence. Prophylaxis. Treatment. 


\section{REFERÊNCIAS BIBLIOGRÁFICAS}

1. AMERICAN PUBLIC HEALTH ASSOCIATION. Communicable Disease in Man. Fifteenth edition, 1990.

2. ANDRADE, J. G; ANDRADE,A.L.S.S; ARAUJO,E.S.O.; OLIVEIRA, R.M.; SILVA,S.A; MARTELLI, C.M.T. \& ZICKER,F. Randomized clinical trial with high dose of chloroquine for treatment of Plasmodium falciparum malaria in Brazil. Rev Inst Med Trop São Paulo ;34(5):467-473.1992.

3. BASCO,L.K \& LE BRAS J. Reversal of chloroquine resistance with desipramine in isolates of Plasmodium falciparum, from Central and West Africa. Trans R Soc Trop Med Hyg ;84:479-481.1990

4. BUNNAG,D. Clinical trials of mefloquine with tetracycline. Southeast Asian $\mathbf{J}$ Trop Med Public Health 23:377- 382.1992.

5. BUNNAG,D. Fansimef for prophylaxis of malaria: a double-blind randomized placebo controled trial. Southeast Asian J Trop Med Public Health ;23:777782.1992

6. CHIODINI,P.L; SOMERVILLE,M; SALAM,I.; TUBBS,H.R.; WOOD,M.J.; ELLIS,C.J.; Exchange transfusion in severe falciparum malaria. Trans R Soc Trop Med Hyg ; 79:865-866.1985.

7. ESPOSITO, R.; ANTONIORI, S.; ORLANDO, G.; RIZARDINI, Z; SPAZZOLINI,A. \& GALLI,M. Exchange transfusion for malaria [letter]. Lancet ;335:790-791.1990.

8. KARBWANG, J.; BANGCHANG,K.N.; THANAVIBUL,A.; BUNNANG,D.; CHONSUPHAJAISIDDHI, T. \& HARINASUTA,T. Comparison of oral artemether and mefloquine in acute uncomplicated falciparum malaria. Lancet ;340:1245-1248.1992.

9. KARBWANG,J. et al. Preliminary report: a comparative clinical trial of artemether and quinine in severe falciparum malaria. Southeast Asian J Trop Med Public Health ;23:768-772.1992.

10. KILE,D.E; ODULA,A.M.J; MARTIN,S.K \& MILHOUS,W.K. Plasmodium falciparum: modulation by calcium antagonists of resistance to chloroqui.., desethylchloroquine, quinine and quinidine in vitro. Trans R Soc Trop Med Hyg ;84:474-478.1990.
11. KREMSNER, P G; ZOTTER, G. M; FELDMEIER,H; BIENZLE,U.; JANSENROSSECK; GRANINGER,W. ROCHA,R.M. \& WERNSDORFER,W.H. Differences in drug response of Plasmodium falciparum within an area of the Amazon region. Rev Inst Med Trop São Paulo ;83:158-161.1989.

12. MALARIA ACTION PROGRAME. World malaria situation 1985 . World Health Statist Quart(WHO) 40:142- 170.1987.

13. MINISTÉRIO DA SAÚDE.Fundação Nacional de Saúde. Manual de terapêutica da malária, 1990.

14. NEIFER,S. \& KREMSNER,P.G. Drug susceptibility of Plasmodium falciparum in the western amazon region, state of Acre, Brazil. Rev Inst Med Trop São Paulo ;33:205-211.1991.

15.ODUOLA,A.M.J.; $\quad$ MOYOU-SOMO,R.S.; KYLE,D.E; $\quad$ MARTIN,S.K.; GERENA,L \& MILHOUS,W.K.; Chloroquine resistant Plasmodium falciparum in indigenous residents of Cameroon. Trans R Soc Trop Med Hyg ;83:308-310.1989.

16.ODUOLA,A.M.J.; SALAKO,L.A.; MILHOUS,W.K.; WALKER,O. \& DESJARDINS,R.E. Reduced in-vitro susceptibility to mefloquine in West African isolates of Plasmodium falciparum. Lancet ;ii:1304-1305.1987.

17.PAYNE,D. Spread of chloroquine resistance in Plasmodium falciparum. Parasitology Today ;3(8):241-246.1987.

18. SOUZA, J. M. Plasmodium falciparum strain resistance to drugs. New drug trials Rev Inst Med Trop São Paulo;34(sup 9):S10-S15.1992.

19. TER KUILE, F. O; DOLAN, G.; NOSTEN, F.; EDSTEIN, M. D; LUXEMBURGER,C; PHAIPUM, L.; CHONGSUPHAJAISIDDHI,T WEBSTER,H.K. \& WHITE,N.J. Halofantrine versus mefloquine in treatment of multidrug-resistant falciparum malaria. Lancet ;341:1044-1049.1993.

20. THAITHONG ,S ; SUEBSAENG,L.; ROONEY,W. \& BEALE,G.H. Evidence of increased chloroquine sensitivity in Thai isolates of Plasmodium falciparum. Trans R Soc Trop Med Hyg ;88:37-38.1988.

21.VALERO,M.V; AMADOR,A.L.R.; GALINDO,C.; FIGEROA,J.; BELLO,M.S.; MURILLO, L.A; MORA,A.L. PATARROYO,G.; ROCHA,C.L.; APONTE,J.J.; SARMIENTO,L.E; LOZADA,D.M. CORONELL,C.G.; ORTEGA,M.N.; ROSAS,J.E.; ALONSO,P.L.; PATARROYO,M.E. Vaccination with SP166, a 
\begin{tabular}{lllllllll}
\hline ANDRADE,J.G. Terapêutica & e & Profilaxia & da & Malária.Revisão.Rev.Pat.Trop.23 & ( 2 & ):181-
\end{tabular} 190,jul./dez.1994

chemically synthetised vaccine, against P.falciparum malaria in Colombia. Lancet ;341:705-710.1993.

22.WARREL,D.A; LOOAREESUWAN,S.; WARELL,M.J.; KASEMSARN,P.; INTRAPRASERT,R.; BUNNAG,D. \& HARINASUTA,T. Dexamethasone proves deleterious in cerebral malaria: a double-blind trial in 100 comatose patients. N Engl J Med ;306:313-319.1982.

23.WATT,G.; LOESUTTIVIBOOL,L.; SHANKS,G.D.; BOUDREAU,E.F.; BROWN,A.E; PANAVADA,K.; WEBSTER, H.K.; \& WECHGRITAYA,S. Quinine with tetracycline for treatment of drug-resistant falciparum malaria in Thailand. Am J Trop Med Hyg ;47(1):108-111.1992.

24.WEINKE, T.; LOSCHER,T.; FLEISCHER,K.; KRETSCHMER,H.; POHLE,H.D.; KOHLER,B.; SCHLUNK,T.; CLEMENS,R. \& BOCK,H.L. The efficacy of halofantrine in the treatment of acute malaria in nonimmune travellers. Am $\mathbf{J}$ Trop Med Hyg ;47:1-5.1992.

25.WHITE,J.W.; WALKER,D.; CRAWLEY,J.; NOSTEN,F.; CHAPMAN,D.; BREWSTER,D. \& GREENWOOD,B.M. Comparison of artemether and chloroquine for severe malaria in Gambian children. Lancet;339:317-321.1992.

26.WHITE, N. J.; WARREL, D. A.; CHANTHAVANICH, P.; LOOAREESUWAN,S.; WARREL,S.; WILLIAMSON,D.H. \& TURNER,R.C. Severe hypoglycaemia and hyperinsulinemia in falciparum malaria. N Engl J Med ;309:61-66.1983.

27.WHO/PAHO/Instituto de Inmunologia. Informal consultation on: Future implementation of malaria vaccines, Bogotá, September - Workshop Report.1993.

28.WIRIMA,J.; MOLYNEUX,M.E.; KHOROMANA,C.; GILLES,H.M. Clinical trials with halofantrine hydrochloride in Malawi. Lancet ;ii:250-252.1988.

29.WYLER,D.J. Malaria: Overview and Update. Clinic Infect Dis ;16:449-458.1993. 